\title{
Successful Retrograde Recanalization of a Very Rare Anomalous Origin Right Coronary Artery Chronic Total Occlusion
}

\section{Gasparini $\mathrm{GL}^{1 *}$, Oreglia $\mathrm{JA}^{2}$, and Reimers $\mathrm{B}^{1}$}

${ }^{1}$ Department of Invasive Cardiology, Istituto Clinico Humanitas, Rozzano (Milan), Italy

${ }^{2}$ Department of Invasive Cardiology, Niguarda Hospital, Milan, Italy

\begin{abstract}
Chronic total coronary occlusions (CTO) still remain one of the most technically challenging clinical scenarios in which to perform interventions. Although the antegrade approach is the most common method of CTO recanalization, a retrograde attempt improves the success rate and its usage has been increasingly adopted in the recent years. Furthermore, abnormalities such as anomalous origin coronary arteries represent other important technically challenging cases for interventional cardiologist. In this case, we describe a rare case of a CTO in the mid portion of an anomalous right coronary artery (RCA), that originated from a high anterior takeoff and progressed in the downward direction, where retrograde approach has been used to overcome the absence of antegrade guiding catheter support.
\end{abstract}

\section{Introduction}

Treatment of CTO lesions remains a major challenge for interventional cardiology and is a frequent reason for a patient's referral to coronary artery bypass surgery (CABG) [1]. Among CTO's angiographic characteristics, anomalous origin is another important angiographic feature that may negatively affect procedural success, due to prevention of guiding catheter engagement into coronary artery, essential to obtain optimal support [2]. Coronary artery anomalies have been found in $1 \%$ of the general population who underwent diagnostic coronary angiography [3]. Coronary intervention for an anomalous coronary artery is a technically challenging and extremely complicated procedure, especially in case of transradial approach for CTOs without a stump $[4,5]$. In the presence of a proximal take off side branch, the anchor technique which uses balloon inflation in a non-target side branch, could be employed to improve guiding catheter support and complete the procedure by antegrade approach [6]. Moreover, when anchoring technique is not feasible, retrograde approach via septal or epicardial collateral channels (CC) may be used to increase rate of PCI success [7]. We describe a rare case of a CTO in the mid portion of an anomalous right coronary artery (RCA), that originated from a high anterior takeoff and progressed in the downward direction, where retrograde approach has been used to overcome the absence of antegrade guiding catheter support.
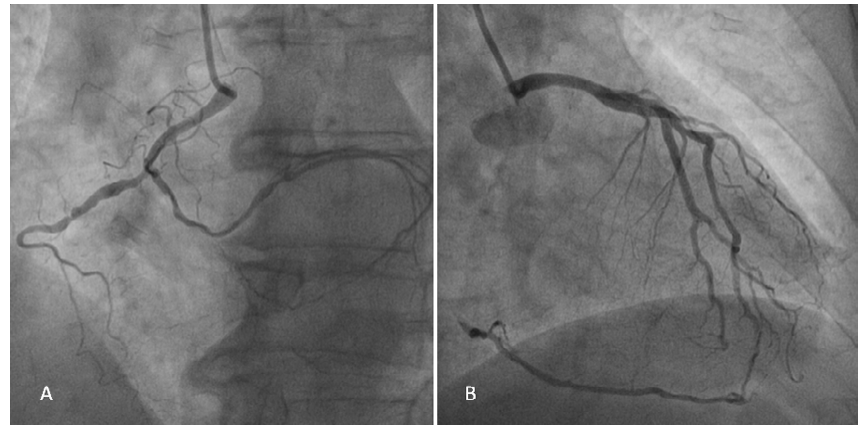

Figure 1: (A) Coronary angiography reveled the RCA originated from a high anterior takeoff and progressed in the downward direction and a CTO without stump just after a small acute marginal branch, in the mid portion of RCA. (B) The circumflex artery (that originated from proximal portion of the RCA) and the LAD did not exhibit significant stenosis. The PDA filled from CC1 epicardial channel from distal LAD.
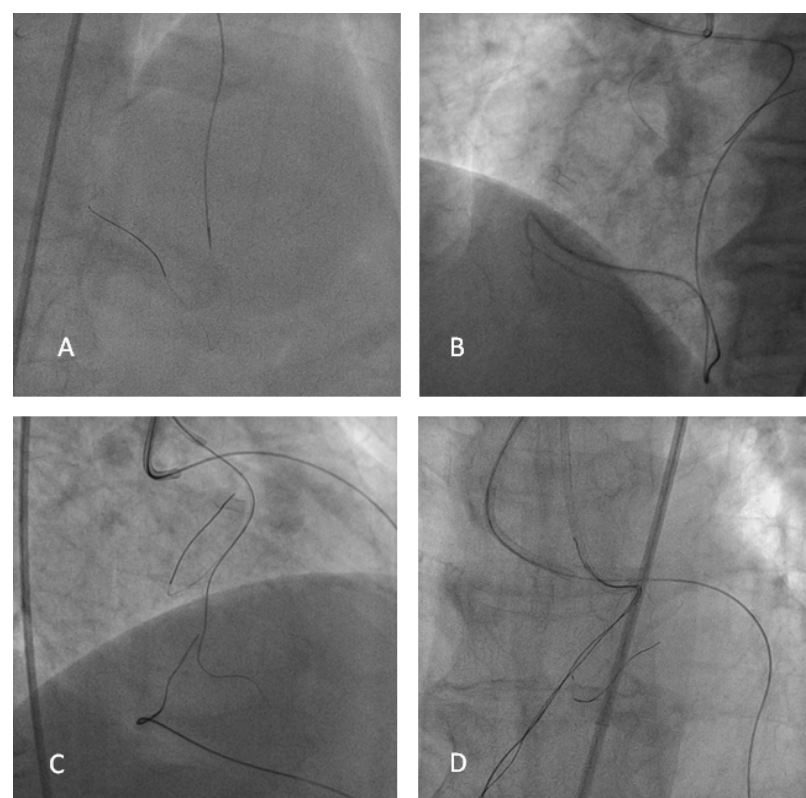

Figure 2: (A) Retrograde approach attempted through the epicardial CC from distal LAD. (B) After successful guidewire CC crossing, a Corsair microcatheter was advanced into PDA and positioned near the distal end of the occlusion. (C) Advancement of an antegrade guidewire close to the proximal cap of the occlusion as a landmark for the retrograde guidewire. (D) After successful CTO crossing, the retrograde guidewire was advanced and trapped into the antegrade guiding catheter.

*Corresponding author: Gasparini GL, Department of Invasive Cardiology, Instituto Clinico Humanitas, Via Manzoni 56, 20089 Rozzano (Milan), Italy, Tel: +390282245996; Fax: +390282243690; E-mail: gabriele_luigi.gasparini@humanitas.it

Received February 16, 2017; Accepted March 16, 2017; Published March 21, 2017

Citation: Gasparini GL, Oreglia JA, Reimers B (2017) Successful Retrograde Recanalization of a Very Rare Anomalous Origin Right Coronary Artery Chronic Total Occlusion. J Cardiovasc Dis Diagn 5: 263. doi: 10.4172/2329-9517.1000263

Copyright: ( 2017 Gasparini GL, et al. This is an open-access article distributed under the terms of the Creative Commons Attribution License, which permits unrestricted use, distribution, and reproduction in any medium, provided the original author and source are credited. 


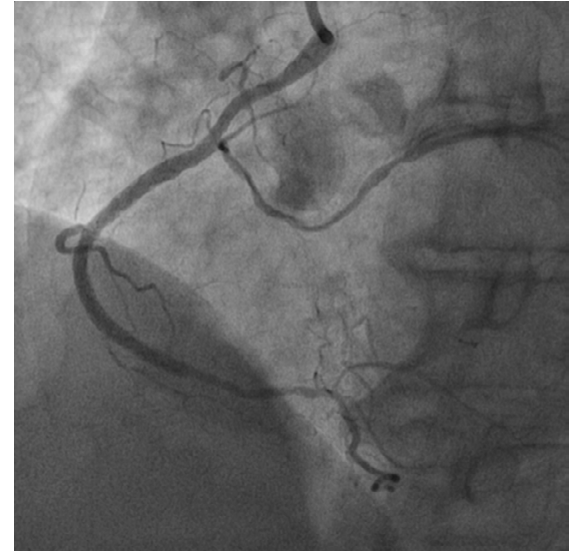

Figure 3: Final coronary angiogram showed an excellent $\mathrm{PCl}$ result.

\section{Case Presentation}

A 65-year-old man with new onset effort angina was referred to our institute to undergo coronary intervention. His coronary risk factors included hypertension, hypercholesterolemia and smoking. Previous coronary angiography revealed the RCA originated from a high anterior takeoff and progressed in the downward direction-a very rare anomalous origin and a CTO which was characterized by absence of a stump just after a small acute marginal branch, in the mid portion of RCA (Figure 1A). The circumflex artery, that originated from proximal portion of the RCA, and the left anterior descending artery (LAD) did not exhibit significant stenosis. The posterior descending artery (PDA) filled from Werner classification CC1 epicardial CC from distal LAD (Figure 1B). The only way to engage the anomalous origin of the RCA was from right radial access, so we used double access with a 6 Fr sheath from the right radial artery and a $45 \mathrm{~cm}$ long $7 \mathrm{Fr}$ sheath from the right femoral artery. RCA was engaged with a 6 Fr Internal Mammary SH guiding catheter, while left coronary artery was engaged with a 7 Fr $90 \mathrm{~cm}$ long XB $3.5 \mathrm{SH}$ guiding catheter. The antegrade approach with the IM guiding catheter was providing poor support, therefore, a balloon-anchoring technique was performed inflating a Maverick $2.0 \times$ $15 \mathrm{~mm}$ balloon up to $12 \mathrm{~atm}$, in the circumflex artery that originated from proximal RCA. However, few minutes after the inflation of the anchor balloon the patient developed angina, so the antegrade approach was aborted and a retrograde approach was attempted through the epicardial CC (Figure 2A). After successful CC crossing with a Sion guidewire, a Corsair microcatheter was advanced into PDA and positioned near the distal end of the occlusion (Figure 2B). Then we advanced an antegrade guidewire close to the proximal cap of the occlusion as a landmark for the retrograde guidewire (Figure 2C). Retrograde Sion guidewire was exchanged with an Ultimate Bros 3 that was able to cross the mid RCA CTO lesion, reaching the proximal true lumen of RCA. Proper position of the retrograde guidewire in the true lumen was confirmed by antegrade IVUS examination. The retrograde wire was then advanced and trapped into the antegrade guiding catheter to provide sufficient backup for Corsair advancement (Figure 2D). Retrograde guidewire trapping into RCA guiding catheter was accomplished by a $2.5 \times 15 \mathrm{~mm}$ monorail balloon inserted antegradely with no guide wire support. Once the Corsair crossed the CTO body and advanced into the antegrade guiding catheter, retrograde Ultimate Bros 3 guidewire was exchanged with a $330 \mathrm{~cm}$ long RG3 wire that was successfully externalized through the opposite hemostatic valve. After externalization of RG3 guidewire, multiple dilatations by antegrade balloons with $1.5 \times 20 \mathrm{~mm}, 2.0 \times 20 \mathrm{~mm}$ were performed. Finally, implantation of three drug eluting stents by overlapping from the distal part of RCA to the proximal part respectively $3.0 \times 12 \mathrm{~mm}, 3.0 \times 31 \mathrm{~mm}$ and $3.5 \times 31 \mathrm{~mm}$ was accomplished and post-dilatation executed by 3.5 $\times 12 \mathrm{~mm}$ NC balloon with excellent angiographic result (Figure 3 ). The patient was discharged two days after the procedure.

\section{Discussion}

Coronary CTO remains one of the most challenging lesion subsets in interventional cardiology, even with the development of medical devices and operator expertise. Anomalous origin is an important angiographic feature that may negatively affect procedural success, due to prevention of guiding catheter engagement into coronary artery. Retrograde approach via septal or epicardial branches may be used to increase rate of PCI success. Knowledge of the specialized devices and techniques and awareness for possible complications could allow successful implementation of these techniques with high procedural success and low complication rates. Operators should be proficient in antegrade CTO PCI before performing a retrograde approach. Retrograde wiring through epicardial collaterals should be reserved until after enough experience has been gained with septal collaterals.

\section{Conclusion}

This case of successful PCI CTO of a very rare anomalous origin RCA underlines how the retrograde approach is a safe and effective alternative in the treatment of CTO, above all in those cases where an anomalous origin of the coronary artery could cause a poor support of the antegrade guiding catheter and the anchoring balloon technique is not feasible.

\section{References}

1. Stone GW, Kandzari DE, Mehran R, Colombo A, Schwartz RS, et al. (2005) Percutaneuos recanalization of chronically occluded coronary artery: a consensus document: Part I. Circulation 112: 2364-2372.

2. Sianos G, Werner GS, Galassi AR, Papafaklis MI, Escaned J, et al. (2012) EuroCTO Club. Recanalisation of chronic total coronary occlusions: 2012 consensus document from the EuroCTO club. Euro Interv 8: 139-145.

3. Angelini P, VBalasco JA, and Flamm S (2002) Coronary anomalies: Incidence, pathophysiology, and clinical relevance. Circulation 105: 2449-2454.

4. Kaneda H, Takahashi S, and Saito S (2007) Successful coronary intervention for chronic total occlusion in an anomalous right coronary artery using the retrograde approach via a collateral vessel. J invasive Cardiol 19: E1-E4.

5. Hsiu-Yu F, Cheng-Ching W, Chiung-Jen W (2009) Successful transradia antegrade coronary intervention of a rare right coronary artery high anterio downward takeoff anomalous chronic total occlusion by double-anchoring technique and retrograde guidance. Int Heart 50: 531-538.

6. Hirokami M, Saito S, and Muto H (2006) Anchoring technique to improve guiding catheter support in coronary angioplasty of chronic total occlusions. Catheter Cardiovasc Interv 67: 366-371

7. Ochiai M (2007) Retrograde approach for chronic total occlusion: present status and prospects. Euro Interv 3: 169-173. 